\title{
SÍNTESE E AVALIAÇÃO DA ATIVIDADE FITOTÓXICA DE LACTONAS DERIVADAS DO 2,4-DIMETIL-8- OXABICICLO[3.2.1]-OCT-6-EN-3-ONA
}

Luiz Cláudio de Almeida Barbosa* e Célia Regina Álvares Maltha

Departamento de Química, Universidade Federal de Viçosa, 36571-000 Viçosa - MG

Eduardo Euclides Lima Borges

Departamento de Engenharia Florestal, Universidade Federal de Viçosa, 36571-000 Viçosa - MG

Recebido em 7/11/00; aceito em 25/7/01

\begin{abstract}
SYNTHESIS AND PHYTOTOXIC ACTIVITY EVALUATION OF LACTONE DERIVATIVES OF 2,4-DIMETHYL-8OXABICYCLO[3.2.1]-OCT-6-EN-3-ONE. The alkene 2,4-dimethyl-8-oxabicyclo[3.2.1]-oct-6-en-3-one (3) was converted to 1,3,10trimethyl-8-oxabicyclo[5.3.0]-dec-3-ene-2,9-dione (7) and 1,3-dimethyl-8-oxabicyclo[5.3.0]-dec-3-ene-2,9-dione (8) with a 55\% overall yield in both cases. Lactones (7) and (8) were converted in two steps to 1,3,4-trimethyl-13-methylene-6-oxatricyclo[8.3.0.0.0.7]trideca-2,5,12-trione (12) (63\%) and 1,3-dimethyl-13-methylene-6-oxatricycle[8.3.0.0 $0^{3,7}$-trideca-2,5,12-trione (13) (45\% from 8). The effect of lactones (7), (8), (12), (13) and the intermediates (5) and (6), at the concentration of $250 \mu \mathrm{g} \mathrm{mL}^{-1}$, on the growth of Cucumis sativus L. and Sorghum bicolor L. was evaluated. The best results were observed for lactone (13) that caused $100 \%$ inhibition on the root growth of C. sativus and lactone (12) that inhibited $90 \%$ of the root growth for S. bicolor.
\end{abstract}

Keywords: [3+4] cycloaddition; lactones; herbicides.

\section{INTRODUÇÃO}

Diversos compostos biologicamente ativos de uso difundido em nossa sociedade, tiveram sua gênese a partir de um produto natural de origem vegetal, animal ou microbiana. Particularmente, os produtos naturais oriundos de plantas podem ser uma potencial fonte de novos agroquímicos, uma vez que elas representam uma fonte inesgotável de substâncias biologicamente ativas ${ }^{1,2}$.

Lactonas sesquiterpênicas são compostos de grande ocorrência na natureza e representam um importante grupo de metabólitos secundários da família Asteraceae ${ }^{3}$. Devido ao seu amplo espectro de atividade biológica ${ }^{4}$, as lactonas sesquiterpênicas constituem uma classe de substâncias com potencial para utilização na medicina, destacando-se as atividades citotóxica e antitumoral ${ }^{5}$, antibacteriana ${ }^{6}$, antiinflamatória ${ }^{7}$, esquistossomicida ${ }^{8}$, antimalárica ${ }^{9}$ e antifúngica ${ }^{6}$.

No setor da agricultura as lactonas sesquiterpênicas também apresentam grande potencial de aplicabilidade ${ }^{4,10}$. Alguns metabólitos secundários podem afetar a germinação e o crescimento de algumas espécies de plantas, embora a natureza e extensão dos efeitos produzidos dependam de vários fatores, dentre eles a estrutura da lactona testada, sua concentração e a espécie na qual ela irá atuar ${ }^{6}$. Esse efeito denomina-se alelopatia ${ }^{3}$, e pode ser de grande importância na agricultura para o controle de plantas daninhas, desde que se estabeleça uma correta associação entre as espécies produtoras de substâncias alelopáticas e as espécies cultivadas.

O conhecimento do mecanismo da atividade biológica destes compostos, bem como a potencialização da atividade dos mesmos, certamente evolui com a síntese e ensaios biológicos de novos compostos que sejam análogos estruturais da molécula considerada modelo ou protótipo.

Uma metodologia sintética de grande versatilidade para a construção do esqueleto sesquiterpênico, do tipo pseudoguaianolídeos, utiliza a reação de cicloadição [3+4] entre um cátion oxialílico (fon-

\footnotetext{
*e-mail: lcab@ufv.br
}

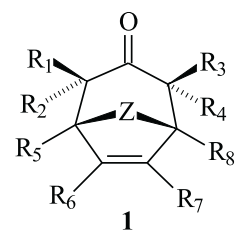

$$
\begin{aligned}
& \mathrm{Z}=\mathrm{O}, \mathrm{CH}_{2}, \mathrm{NR}^{\prime} \\
& \mathrm{R}=\mathrm{H}, \text { grupos alquil ou aril }
\end{aligned}
$$

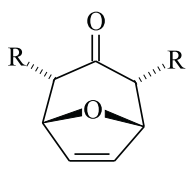

$2 \mathrm{R}=\mathrm{H}$

$3 \quad \mathrm{R}=\mathrm{CH}_{3}$ te de três carbonos) e um dieno (unidade com quatro carbonos), que permite a obtenção direta de carbociclos de sete membros ${ }^{11}$. As reações de cicloadição [3+4] geram cicloadutos com estrutura genérica do tipo 1, com grande potencial para modificações químicas.

Diversos compostos derivados do 8-oxabiciclo[3.2.1]-oct-6-en3-ona (2) e do 2,4-dimetil-8-oxabiciclo[3.2.1]-oct-6-en-3-ona (3), com potencial atividade herbicida e/ou reguladora sobre o crescimento de plantas, foram preparados anteriormente ${ }^{12,13,14}$. Apresentamos neste trabalho a síntese de novas lactonas (12 e 13) derivadas do 2,4-dimetil-8-oxabiciclo[3.2.1]-oct-6-en-3-ona (3). Essas lactonas (12 e 13) assemelham-se estruturalmente aos pseudoguaianolídeos, que são lactonas sesquiterpênicas que possuem uma ampla diversidade de efeitos biológicos, incluindo o efeito sobre o crescimento de plantas ${ }^{6}$. Também são apresentados os resultados dos ensaios biológicos realizados com Sorghum bicolor L. e Cucumis sativus L.

\section{PARTE EXPERIMENTAL}

\section{Procedimentos Experimentais Gerais}

As temperaturas de fusão foram determinadas em aparelho Kofler R Winkel Sotting, modelo 17717 e foram corrigidas. Os espectros na região do infravermelho foram registrados em pastilhas de $\mathrm{KBr}$ ou em solução de clorofórmio, em um espectrômetro Perkin Elmer FTIR 1000, na região de 4000 a $600 \mathrm{~cm}^{-1}$. Os espectros de RMN de ${ }^{1} \mathrm{H}$ foram obtidos em espectrômetro Perkin Elmer R34 (220 MHz) ou em aparelho Bruker WM400 (400 MHz). Os deslocamentos quí- 
micos estão apresentados em partes por milhão $(\delta)$ relativos ao tetrametilsilano (TMS, $\delta=0,0$ ), e como solvente utilizou-se $\mathrm{CDCl}_{3}$. Os dados de nOe foram obtidos por diferença espectral, subtraindose os espectros registrados com irradiação nas frequências de ressonância dos átomos de hidrogênio daqueles obtidos com irradiação em região livre de ressonância. Os espectros de massas foram obtidos em espectrômetro V.G. Analytical ZAB-IF, operando a 70eV.

Os reagentes utilizados nas reações e os solventes utilizados em cromatografia em coluna de sílica gel foram previamente purificados e secados de acordo com os procedimentos descritos na literatu$\mathrm{ra}^{15}$. Para a purificação dos compostos utilizou-se a cromatografia em coluna de sílica gel 60 (70-230 mesh-ASTM, Merck). Todas as reações foram monitoradas por cromatografia em camada delgada utilizando-se placas de sílica gel Camlab-Polygram SILK/UV ${ }_{254}$, com $1,25 \mathrm{~mm}$ de espessura.

O composto (3) foi preparado de acordo com a metodologia descrita na literatura ${ }^{12,18}$.

\section{Procedimentos Sintéticos}

$2 \alpha, 4 \alpha$,-dimetil-8-oxabiciclo[3.2.1]-oct-3-ona (4). A uma solução do alqueno (3) $(9,8 \mathrm{~g}, 64,4 \mathrm{mmol})$ em $200 \mathrm{~mL}$ de acetato de etila foi adicionado $0,9 \mathrm{~g} \mathrm{de} \mathrm{Pd} / \mathrm{C} 10 \%$. A mistura foi submetida à hidrogenação durante 18 horas em um hidrogenador Parr, a uma pressão de 3 atm de $\mathrm{H}_{2}$, e em seguida filtrada sob Celite. $\mathrm{O}$ filtrado foi concentrado em evaporador rotatório fornecendo o composto (4) (óleo claro, 9,9 g, 64,2 mmol, 100\%). IV (filme $\mathrm{NaCl}, \mathrm{cm}^{-1}$ ) $\bar{v}_{\text {max }}: 2980,2880,1708$, 1381, 1159, 1047, 951. RMN de ${ }^{\mathbf{1}} \mathbf{H}\left(\mathrm{CDCl}_{3}, 400 \mathrm{MHz}\right) \delta: 0,95$ (d, $6 \mathrm{H}, J=7 \mathrm{~Hz}, 2 \times \mathrm{Me}$ ), 1,70-1,90 (m, 4H, H6, H7), 2,60-3,00 (m, 2H, $\mathrm{H} 2$, H4), 4,50 (m, 2H, H1, H5). EM m/z (\%): 154,096 (M+, $\mathrm{C}_{9} \mathrm{H}_{14} \mathrm{O}_{2}$ requer 154,099, 15), 98 (20), 69 (40), 56 (100), 41 (40).

$2 \alpha, 4 \alpha$-dimetil-2 $\beta$-(1-metoxicarboniletil)-8-oxabiciclo[3.2.1]-oct3-ona (5). A uma solução de N,N-diisopropilamina $(2,85 \mathrm{~mL}, 20,2$ mmol) em tetraidrofurano $(40 \mathrm{~mL})$ a $-10{ }^{\circ} \mathrm{C}$, sob atmosfera de nitrogênio, foi adicionado butil lítio $\left(12,7 \mathrm{~mL}\right.$, de uma solução $1,6 \mathrm{~mol} \mathrm{~L}^{-1}$, em hexano, 20,2 mmol). A solução foi mantida sob agitação magnética por 30 minutos, sendo resfriada a $-78^{\circ} \mathrm{C}$. Em seguida adicionaramse o oxabiciclo (4) $(2,0 \mathrm{~g}, 12,99 \mathrm{mmol})$, em $20 \mathrm{~mL}$ de tetraidrofurano e $20 \mathrm{~mL}$ de N,N'-dimetil-N,N'-propileno uréia (DMPU). Após 1,5 horas adicionou-se $( \pm)$-2-bromopropionato de metila $(5,8 \mathrm{~mL}, 8,68 \mathrm{~g}$, $52 \mathrm{mmol}$ ). A mistura foi mantida sob agitação magnética, à temperatura ambiente por 16 horas. Após decorrido esse tempo, adicionaramse $60 \mathrm{~mL}$ de água e o produto foi extraído com acetato de etila $(5 \times 50$ $\mathrm{mL})$. A fase orgânica foi lavada com solução saturada de $\mathrm{NaCl}(2$ x 40 $\mathrm{mL})$, secada sobre $\mathrm{MgSO}_{4}$ e concentrada em evaporador rotatório. Obteve-se um óleo alaranjado, cuja purificação por cromatografia em coluna de sílica gel (éter de petróleo:éter dietílico 2:1) forneceu o ceto éster (5) (2,24 g, 9,33 mmol, 72\%). $\mathrm{T}_{\mathrm{f}}=49-50{ }^{\circ} \mathrm{C}$, sólido branco, IV $\left(\mathrm{KBr}, \mathrm{cm}^{-1}\right) \overline{\mathrm{v}}_{\max }: 2950,2893,1735,1708,1451,1254,1080,1052$, 874, 729. RMN de ${ }^{1} \mathbf{H}\left(\mathrm{CDCl}_{3}, 400 \mathrm{MHz}\right) \delta: 0,90\left(\mathrm{~d}, 3 \mathrm{H}, J_{\mathrm{Me}, 4}=7,2\right.$ $\mathrm{Hz}, 4-\mathrm{Me}), 0,95$ (d, 3H, $J_{\mathrm{Me}, 2}=7,2 \mathrm{~Hz}, 2$ '-Me), 0,92 (s, 3H, 2-Me), 1,60-2,00 (m, 4H, H6 e H7), 2,90 (quinteto largo, 1H, $J \cong 7,2 \mathrm{~Hz}, \mathrm{H} 4$ ), 3,58 (q, 1H, $\left.J_{2^{\prime}, \mathrm{Me}}=7,2 \mathrm{~Hz}, \mathrm{H} 2^{\prime}\right), 3,75$ (s, 3H, O-Me), 4,45-4,55 (m, $2 \mathrm{H}, \mathrm{H} 1$ e H5). RMN de ${ }^{13} \mathbf{C}\left(\mathrm{CDCl}_{3}, 100 \mathrm{MHz}\right) \delta$ : 9,93 (Me-2'), 11,97 (Me-4), 12,26 (Me-2), 24,65 (C7), 25,01 (C6), 41,84 (C4), 47,40 (C2'), 51,70 (C2), 56,98 (O-CH $)$, 81,36 (C1), 81,57 (C5), 173,84 (C1'), 210,61 (C3). Análise elementar: Encontrado C 64,99\%, H 8,51\%; $\mathrm{C}_{13} \mathrm{H}_{20} \mathrm{O}_{4}$ requer C 64,96\%, $\mathrm{H} \mathrm{8,39 \% .}$

$2 \alpha, 4 \alpha$,-dimetil-2 $\beta$-(metoxicarbonilmetil)-8-oxabiciclo[3.2.1]-oct3-ona (6). A uma solução de N,N-diisopropilamina $(2,85 \mathrm{~mL}, 20,2$ mmol) em tetraidrofurano $(40 \mathrm{~mL}) \mathrm{a}-10{ }^{\circ} \mathrm{C}$, sob atmosfera de nitrogênio, foi adicionado butil lítio $\left(12,7 \mathrm{~mL}\right.$, de uma solução $1,6 \mathrm{~mol} \mathrm{~L}^{-1}$, em hexano, 20,2 mmol). A solução foi mantida sob agitação magnéti- ca por 30 minutos, sendo resfriada a $-78^{\circ} \mathrm{C}$. Em seguida adicionaramse o oxabiciclo (4) $(1,90 \mathrm{~g}, 12,33 \mathrm{mmol})$, em $20 \mathrm{~mL}$ de tetraidrofurano e também $20 \mathrm{~mL}$ de N,N'- dimetil-N,N'-propileno uréia (DMPU). Após 1,5 horas adicionou-se rapidamente bromoacetato de metila (2,6 $\mathrm{mL}, 4,2 \mathrm{~g}, 30,75 \mathrm{mmol})$. A mistura foi mantida sob agitação magnética, à temperatura ambiente por 18 horas. Após decorrido esse tempo, adicionaram-se $60 \mathrm{~mL}$ de água e o produto foi extraído com acetato de etila $(4 \times 50 \mathrm{~mL})$. A fase orgânica foi lavada com solução saturada de $\mathrm{NaCl}(2$ x $40 \mathrm{~mL})$, secada sobre $\mathrm{MgSO}_{4}$ e concentrada em evaporador rotatório. Obteve-se um óleo alaranjado, cuja purificação por cromatografia em coluna de sílica gel (éter de petróleo:éter dietílico 2:1) forneceu o ceto éster $(6)(2,68 \mathrm{~g}, 11,8 \mathrm{mmol}, 96 \%)$. $\mathrm{T}_{\mathrm{f}}=42-44{ }^{\circ} \mathrm{C}$, sólido branco, IV $\left(\mathrm{KBr}, \mathrm{cm}^{-1}\right) \overline{\mathrm{v}}_{\max }: 2955,2890,1735,1708,1155$, $1055,1028,953,920 . \mathbf{R M N}^{2}{ }^{1} \mathbf{H} \mathbf{H}\left(\mathrm{CDCl}_{3}, 220 \mathrm{MHz}\right) \delta: 0,92(\mathrm{~d}, 2 \mathrm{H}$, $\left.J_{\mathrm{Me}, 4}=6,6 \mathrm{~Hz}, 4-\mathrm{Me}\right), 1,0$ (s, 3H, 2-Me), 1,58-1,92 (m, 4H, H6 e H7), 2,55 e 3,19 (2d, 2H, $J_{\text {gem }}=14,1 \mathrm{~Hz}, \mathrm{H} 2$ ') , 3,04-3, 14 (m, 1H, H4), 3,60 (s, 3H, O-CH $), 4,21\left(\mathrm{~d}, 1 \mathrm{H}, J_{1,7}=7,2 \mathrm{~Hz}, \mathrm{H1}\right), 4,45\left(\mathrm{t}, 1 \mathrm{H}, J_{5,4}=J_{5,6}=\right.$ $5,5 \mathrm{~Hz}, \mathrm{H} 5)$. RMN de ${ }^{13} \mathbf{C}\left(\mathrm{CDCl}_{3}, 55 \mathrm{MHz}\right) \delta: 10,04(\mathrm{Me}-4), 17,06$ (Me-2), 24,69 (C7), 24,99 (C6), 42,85 (C2), 47,36 (C4), 51,93 (C2'), 53,76 (O-Me), 81,88 (C1), 83,57 (C5), 171,71 (C1'), 210,75 (C3). EM m/z (\%): 226,1195 $\left(\mathrm{M}^{+}, \mathrm{C}_{12} \mathrm{H}_{18} \mathrm{O}_{4}\right.$ requer 226,1205, 18), 195 (19), 158 (44), 128 (38), 126 (100), 100 (59), 86 (19), 59 (19), 57 (15), 55 (44). Análise elementar: Encontrado $\mathrm{C} \mathrm{63,63 \% ,} \mathrm{H} \mathrm{8,07 \% ;} \mathrm{C}_{12} \mathrm{H}_{18} \mathrm{O}_{4}$ requer $\mathrm{C} 63,67 \%, \mathrm{H} 8,02 \%$.

1,3,10-trimetil-8-oxabiciclo[5.3.0]-dec-3-eno-2,9-diona (7). A uma solução do ceto éster (5) $(0,6$ g, 2,5 mmol) e iodeto de potássio (1,25 g, 7,5 mmol), em $10 \mathrm{~mL}$ de clorofórmio, foi adicionado $\mathrm{BF}_{3} \cdot \mathrm{Et}_{2} \mathrm{O}$ $(1,84 \mathrm{~mL}, 15 \mathrm{mmol})$ gota a gota, sob agitação magnética e atmosfera de nitrogênio, a $0{ }^{\circ} \mathrm{C}$. Após cessada a adição de $\mathrm{BF}_{3}$. Et ${ }_{2} \mathrm{O}$, a mistura foi mantida sob agitação magnética e aquecimento a $60-65{ }^{\circ} \mathrm{C}$ durante sete dias, quando então adicionaram-se $30 \mathrm{~mL}$ de solução saturada de $\mathrm{NaHCO}_{3}$. Em seguida, foi feita a extração com éter dietílico (5 x 30 $\mathrm{mL}$ ). A fase orgânica foi lavada com $15 \mathrm{~mL}$ de solução $0,5 \mathrm{~mol} \mathrm{~L}^{-1}$ de $\mathrm{Na}_{2} \mathrm{~S}_{2} \mathrm{O}_{3}, 20 \mathrm{~mL}$ de água e secada sobre $\mathrm{MgSO}_{4}$. A evaporação do solvente da fase orgânica resultou em um óleo escuro que, após purificação por cromatografia em coluna de sílica gel (éter de petróleo:éter dietílico 3:2), forneceu a lactona (7) em $55 \%$ de rendimento $(0,30 \mathrm{~g}$, $1,37 \mathrm{mmol}) . \mathrm{T}_{\mathrm{f}}=81-83{ }^{\circ} \mathrm{C}$, sólido branco, IV $\left(\mathrm{KBr}, \mathrm{cm}^{-1}\right) \overline{\mathrm{v}}_{\max }: 3022$, 2978, 2949, 1767, 1677, 1452, 1427, 1383, 1221, 1175, 1134, 1028, 968, 810, 722. RMN de ${ }^{1} \mathbf{H}\left(\mathrm{CDCl}_{3}, 220 \mathrm{MHz}\right) \delta: 1,20\left(\mathrm{~d}, 3 \mathrm{H}, J_{\mathrm{Me}, 10}=\right.$ 7,5 Hz, Me), 1,38 (s, 3H, Me), 1,90 (m, 3H, Me), 1,95-2,05 (m, 2H, H6), 2,35-2,48 (m, 2H, H5), 3,29 (q, 1H, $\left.J_{10, \mathrm{Me}}=7,5 \mathrm{~Hz}, \mathrm{H} 10\right), 4,28$ $\left(\mathrm{dd}, 1 \mathrm{H}, J_{7,6}=8,8, J_{7,6}=3 \mathrm{~Hz}, \mathrm{H} 7\right), 6,15(\mathrm{~m}, 1 \mathrm{H}, \mathrm{H} 4)$. RMN de ${ }^{13} \mathbf{C}$ $\left(\mathrm{CDCl}_{3}, 55 \mathrm{MHz}\right) \delta$ : 10,21 (Me-10), 19,64 (Me-1), 21,85 (C6), 27,25 (C10), 30,24 (C5), 39,34 (Me-3), 60,15 (C1), 84,48 (C7), 134,42 (C3), 135,79 (C4), 177,66 (C9), 205,50 (C2). Análise elementar: Encontrado $\mathrm{C} 69,31 \%, \mathrm{H} 7,80 \% ; \mathrm{C}_{12} \mathrm{H}_{16} \mathrm{O}_{3}$ requer $\mathrm{C} 69,21 \%, \mathrm{H} 7,75 \%$.

1,3-dimetil-8-oxabiciclo[5.3.0]-dec-3-eno-2,9-diona (8) e 1,3dimetil-8-oxabiciclo[4.4.0]-dec-3-eno-2,9-diona (9). A uma solução do ceto éster (6) $(9,1 \mathrm{~g}, 40,2 \mathrm{mmol})$ e iodeto de potássio (19,8 g, 120 $\mathrm{mmol})$, em $60 \mathrm{~mL}$ de clorofórmio, foi adicionado $\mathrm{BF}_{3} \cdot \mathrm{Et}_{2} \mathrm{O}(21,3$ $\mathrm{mL}, 173,3 \mathrm{mmol}$ ) gota a gota, sob agitação magnética e atmosfera de nitrogênio, a $0{ }^{\circ} \mathrm{C}$. Após cessada a adição de $\mathrm{BF}_{3}$. $\mathrm{Et}_{2} \mathrm{O}$, a mistura foi mantida sob agitação magnética e aquecimento a $60-65{ }^{\circ} \mathrm{C}$ durante 10 dias, quando então adicionaram-se $60 \mathrm{~mL}$ de solução saturada de $\mathrm{NaHCO}_{3}$. Em seguida, foi feita a extração com éter dietílico (4 x $80 \mathrm{~mL}$ ). A fase orgânica foi lavada com $30 \mathrm{~mL}$ de solução $0,5 \mathrm{~mol} \mathrm{~L}^{-1} \mathrm{de}$ $\mathrm{Na}_{2} \mathrm{~S}_{2} \mathrm{O}_{3}, 60 \mathrm{~mL}$ de água e secada sobre $\mathrm{MgSO}_{4}$. A evaporação do solvente da fase orgânica resultou em um óleo escuro que, após purificação por cromatografia em coluna de sílica gel (éter de petróleo:éter dietílico 1:1), forneceu a lactona (8) (óleo incolor, 4,29 g, 22,1 mmol, 55\%) e a lactona (9) (óleo incolor, 0,39 g, $2 \mathrm{mmol}, 5 \%)$. 
Dados de (8): IV (filme NaCl, $\mathrm{cm}^{-1}$ ) $\overline{\mathrm{v}}_{\text {max }}: 2932,1782,1675,1452$, 1404, 1348, 1206, 1100, 1066, 871, 800). RMN de ${ }^{\mathbf{1}} \mathbf{H}\left(\mathrm{CDCl}_{3}, 220\right.$ MHz) $\delta$ : 1,42 (s, 3H, 1-Me), 1,88 (dd, 3H, $J_{\mathrm{Me}, 4}=J_{\mathrm{Me}, 5}=1,5 \mathrm{~Hz}, 3-$ Me), 1,80-2,00 (2m, 2H, H6 e H6'), 2,30 (d, 1H, $\left.J_{\text {gem }}=18 \mathrm{~Hz}, \mathrm{H} 10\right)$, 2,30-2,60 (m, 2H, H5), 3,36 (d, 1H, $J_{\text {gem }}=18 \mathrm{~Hz}, \mathrm{H} 10$ '), 4,42 (dd, $1 \mathrm{H}$, $\left.J_{7,6}=10 \mathrm{~Hz}, J_{7,6}=3 \mathrm{~Hz}, \mathrm{H} 7\right), 6,20(\mathrm{~m}, 1 \mathrm{H}, \mathrm{H} 4)$. RMN de ${ }^{13} \mathbf{C}\left(\mathrm{CDCl}_{3}\right.$, $55 \mathrm{MHz}) \delta$ : 21,48 (C6), 25,01 (1-Me), 26,27 (C10), 31,13 (C5), 38,04 (3-Me), 57,20 (C1), 86,07 (C7), 135,66 (C3), 136,02 (C4), 173,84 (C9), 204,13 (C2). EM m/z (\%): 194,0940 (M+, $\mathrm{C}_{11} \mathrm{H}_{14} \mathrm{O}_{3}$ requer 194,0943, 83) , 150 (22), 111 (35), 108 (30), 99 (16), 98 (36), 95 (100), 82 (99), 71 (17), 54 (25). Análise elementar: Encontrado C $67,90 \%, \mathrm{H} 7,31 \% ; \mathrm{C}_{11} \mathrm{H}_{14} \mathrm{O}_{3}$ requer $\mathrm{C} 68,02 \%, \mathrm{H} 7,26 \%$.

Dados de (9): IV (filme NaCl, cm ${ }^{-1}$ ) $\overline{\mathrm{v}}_{\text {max }}: 3020,2971,1737,1670$, $1451,1237,1115,1057,1035,980,837,696 . \mathbf{R M N}$ de ${ }^{1} \mathbf{H}\left(\mathrm{CDCl}_{3}\right.$, $220 \mathrm{MHz}) \delta: 1,32$ (s, 3H, 1-Me), 1,81 (m, 3H, 3-Me), 2,18 (d, 1H, $\left.J_{\text {gem }}=18 \mathrm{~Hz}, \mathrm{H} 10\right), 2,27$ e 2,82 (2dm, $J_{\text {gem }} \cong 20 \mathrm{~Hz}, \mathrm{H} 5$ e H5'), 2,50 (m, 1H, H6), 3,26 (d, 1H, $J_{\text {gem }}=18 \mathrm{~Hz}, \stackrel{\mathrm{H} 10}{ }$ '), 4,18 e 4,38 (2dd, 2H, $\left.J_{1}=11,5 \mathrm{~Hz}, J=5 \mathrm{~Hz}, \mathrm{H} 7 \mathrm{e} \mathrm{H}^{\mathrm{H}}{ }^{\prime}\right), 6,60$ (m, 1H, H4). RMN de ${ }^{13} \mathbf{C}$ $\left(\mathrm{CDCl}_{3}, 55 \mathrm{MHz}\right) \delta$ : 15,7 (1-Me), 23,0 (3-Me), 24,1 (C10), 36,1 (C5), 37,3 (C6), 43,1 (C1), 69,7 (C7), 132,5 (C3), 140,3 (C4), 168,5 (C9), 199,5 (C2). Análise elementar: Encontrado C 67,62\%, H $7,32 \% ; \mathrm{C}_{11} \mathrm{H}_{14} \mathrm{O}_{3}$ requer $\mathrm{C} 68,02 \%, \mathrm{H} 7,26 \%$.

1,3,4-trimetil-12-trimetilsililoxi-12-vinil-6-oxatriciclo[8.2.0.0 $\left.0^{3,7}\right]$ dodeca-2,5-diona (10). Uma solução da lactona (7) $(0,33 \mathrm{~g}, 1,58$ mmol) e 2-trimetilsililoxibuta-1,3-dieno (1,0 g, 7,02 mmol), em 50 $\mathrm{mL}$ de dimetoxietano foi colocada em um recipiente de Pyrex e degaseificada usando nitrogênio. A mistura foi então irradiada durante 10 horas com duas lâmpadas de mercúrio (500 Watt), mantendo-se a temperatura de $20{ }^{\circ} \mathrm{C}$. Após a remoção do solvente em evaporador rotatório, foi obtido um resíduo branco. Este material foi purificado por cromatografia em coluna de sílica gel (éter de petróleo: éter dietílico 1:1), e resultou no triciclo (10) $(0,05 \mathrm{~g}, 0,14 \mathrm{mmol}$, $8 \%) . \mathrm{T}_{\mathrm{f}}=150{ }^{\circ} \mathrm{C}$, com decomposição, sólido branco, IV $\left(\mathrm{KBr}, \mathrm{cm}^{-1}\right)$ $\bar{v}_{\max }:$ 3017, 2956, 2870, 1767, 1691, 1604, 1457, 1251, 1027, 931, 841. RMN de ${ }^{1} \mathbf{H}\left(\mathrm{CDCl}_{3}, 220 \mathrm{MHz}\right) \delta: 0,10$ (s, 9H, Si(Me) $\left.)_{3}\right), 1,18$ (s, 3H, 3-Me), 1,37 (s, 3H, 1-Me), 1,28 (d, 3H, $J_{M e, 4}=6,6 \mathrm{~Hz}, 4-$ $\mathrm{CH}_{3}$ ), 1,50-2,50 (m, 7H, H10, H9, H8, H11), 2,68 (q, 1H, $J_{4, \mathrm{Me}}=6,6$ $\mathrm{Hz}, \mathrm{H} 4), 4,45$ (dd, 1H, $\left.J_{7.8}=10,5 \mathrm{~Hz}, J_{7,8}=4 \mathrm{~Hz}, \mathrm{H} 7\right), 5,18$ (dd, $1 \mathrm{H}$, $\left.J_{\mathrm{b}, \mathrm{a}}=10,5 \mathrm{~Hz}, J_{\mathrm{b}, \mathrm{c}}=1 \mathrm{~Hz}, \mathrm{Hb}\right), 5,28\left(\mathrm{dd}, 1 \mathrm{H}, J_{\mathrm{c}, \mathrm{a}}=18 \mathrm{~Hz}, J_{\mathrm{c}, \mathrm{b}}=1 \mathrm{~Hz}\right.$, $\mathrm{Hc}), 5,98\left(\mathrm{dd}, 1 \mathrm{H}, J_{\mathrm{a}, \mathrm{c}}=18 \mathrm{~Hz}, J_{\mathrm{a}, \mathrm{b}}=10,5 \mathrm{~Hz}, \mathrm{Ha}\right) . \mathbf{E M ~} \mathrm{m} / \mathrm{z}(\%)$ : $350,1913\left(\mathrm{M}^{+}, \mathrm{C}_{19} \mathrm{H}_{30} \mathrm{O}_{4} \mathrm{Si}\right.$, requer 350,1913$)$.

1,3,4-trimetil-13-metileno-6-oxatriciclo[8.3.0.0 $\left.0^{3,7}\right]$-trideca2,5,12-triona (12). A uma solução do triciclo (10) $(0,11 \mathrm{~g}, 0,3 \mathrm{mmol})$, em $8 \mathrm{~mL}$ de tetraidrofurano, adicionaram-se $\mathrm{Pd}(\mathrm{PhCN})_{2} \mathrm{Cl}_{2}(0,02 \mathrm{~g}$, $0,04 \mathrm{mmol})$ e $p$-benzoquinona $(0,108 \mathrm{~g}, 1,0 \mathrm{mmol})$. A mistura foi mantida em refluxo, sob atmosfera de nitrogênio e agitação magnética, por 4 horas. Após o resfriamento da mistura à temperatura ambiente adiciaram-se $20 \mathrm{~mL}$ de água e $20 \mathrm{~mL}$ de éter dietílico. As duas fases foram separadas em funil de separação e a fase aquosa foi extraída com éter dietílico $(5 \times 20 \mathrm{~mL})$. A fase orgânica foi reunida e lavada com solução saturada de $\mathrm{NaCl}(2 \times 20 \mathrm{~mL})$, solução $2 \mathrm{~mol} \mathrm{~L}^{-1}$ de $\mathrm{Na}_{2} \mathrm{~S}_{2} \mathrm{O}_{3}(20 \mathrm{~mL})$, secada sobre $\mathrm{MgSO}_{4}$ e concentrada em evaporador rotatório. $\mathrm{O}$ resíduo verde obtido foi purificado por cromatografia em coluna de sílica gel (éter de petróleo:éter dietílico 1:1) para fornecer a lactona tricíclia (12), que foi recristalizada em éter dietílico $(0,05 \mathrm{~g}, 0,19 \mathrm{mmol}, 63 \%) . \mathrm{T}_{\mathrm{f}}=172-175^{\circ} \mathrm{C}$, sólido branco. IV $\left(\mathrm{KBr}, \mathrm{cm}^{-1}\right) \overline{\mathrm{v}}_{\max }: 3019,2930,2871,1768,1729,1692,1633$, 1603, 1492, 1387, 1287, 1261, 1193, 1087, 1044, 992, 877, 710. RMN de ${ }^{1} \mathbf{H}\left(\mathrm{CDCl}_{3}, 400 \mathrm{MHz}\right) \delta: 1,21$ (s, 3H, 3- $\left.\mathrm{CH}_{3}\right), 1,24$ (s, 3H, 1- $\mathrm{CH}_{3}$ ), 1,33 (d, $\left.J_{\mathrm{Me}, 4}=7,2 \mathrm{~Hz}, 4-\mathrm{Me}\right), 1,58-1,77$ (m, 2H, H-8', H9'), 2,20-2,18 (m, 2H, H8, H9), 2,23 (dd, 1H, $J_{11^{\prime}, 11}=17,8 \mathrm{~Hz}, J_{11^{\prime} 10}=$ $13,2 \mathrm{~Hz}, \mathrm{H} 11$ ') , 2,50 (dd, $1 \mathrm{H}, J_{11,11}=17,8 \mathrm{~Hz}, J_{11,10}=7,2 \mathrm{~Hz}, \mathrm{H} 11$ ), 2,60-2,69 ( m, 1H, H10), 3,05 (q, 1H, $\left.J_{4, \mathrm{Me}}=7,2 \mathrm{~Hz}, \mathrm{H} 4\right), 4,44$ (dd, $\left.1 \mathrm{H}, J_{7,8}=11,9 \mathrm{~Hz}, J_{7,8}=1 \mathrm{~Hz}, \mathrm{H} 7\right), 5,34(\mathrm{~s}, 1 \mathrm{H}, \mathrm{Hb}), 5,94(\mathrm{~s}, 1 \mathrm{H}$, $\mathrm{Ha})$. RMN de ${ }^{13} \mathbf{C}\left(\mathrm{CDCl}_{3}, 100 \mathrm{MHz}\right) \delta: 10,9$ (Me-4), 20,3 (Me-3), 20,4 (Me-1), 23,8 (C8), 28,8 (C9), 35,8 (C11), 39,8 (C1), 40,5 (C10), 55,8 (C4), 59,6 (C3), 83,3 (C7), 116,7 (C14), 149,2 (C13), 176,2 (C5), 201,6 (C12), 212,3 (C2). EM m/z (\%): 276,1335 ( $\mathrm{M}^{+}, \mathrm{C}_{16} \mathrm{H}_{20} \mathrm{O}_{4}$ requer 276,1362, 38), 248 (40), 202 (13), 187 (7), 174 (20), 151 (15), 135 (40), 122 (100), 109 (30), 83 (62), 55 (62).

\section{Ensaios Biológicos}

Os ensaios de atividade sobre sementes de Sorghum bicolor L. (sorgo) e Cucumis sativus L. (pepino) foram realizados de acordo com a metodologia descrita na literatura ${ }^{16}$. Foram preparadas soluções dos compostos (5), (6), (7), (8), (12) e (13) em diclorometano, nas concentrações de 100 e $250 \mu \mathrm{g} \mathrm{mL}^{-1}$. Os experimentos foram conduzidos em placas de Petri de $6 \mathrm{~cm}$ de diâmetro, nas quais foram adicionados $2 \mathrm{~mL}$ de cada solução. As placas foram deixadas à temperatura ambiente até a evaporação do solvente. Em seguida foram adicionados $2 \mathrm{~mL}$ de água e 20 sementes da planta teste, previamente esterilizadas por imersão durante 10 minutos em solução a $20 \%$ de hipoclorito de sódio. As placas foram incubadas a $25{ }^{\circ} \mathrm{C}$, sob luz fluorescente $(8 \times 40 \mathrm{~W})$, por um período de três dias, quando então os comprimentos das raízes foram medidos. As porcentagens de inibição foram calculadas com base nos dados obtidos nos experimentos de controle, realizados nas mesmas condições descritas. $\mathrm{O}$ delineamento experimental foi inteiramente casualizado com cinco repetições. Os dados foram analisados utilizando-se o teste de Tukey ${ }^{17}$.

\section{RESULTADOS E DISCUSSÃO}

\section{Síntese}

Em trabalho anterior, descrevemos a síntese do composto $(\mathbf{1 3})^{18}$. Assim, visando preparar o composto (12), análogo de (13) contendo um grupo metila no carbono $\alpha$ à carbonila da lactona, a mesma estratégia sintética foi empregada, conforme apresentado no Esquema 1.

Realizou-se inicialmente a síntese do intermediário (3), através da reação de cicloadição [3+4] entre o cátion oxialílico gerado a partir da 2,4-dibromopentan-3-ona e furano ${ }^{12,18}$. A reação de hidrogenação catalítica do cicloaduto $(3)^{19}$ resultou na obtenção quantitativa do oxabiciclo saturado (4). O tratamento do composto (4) com diisopropilamideto de lítio (LDA) resultou na formação do enolato correspondente, o qual foi tratado com excesso de bromoacetato de metila ou com uma mistura racêmica de 2-bromopropionato de metila (Esquema 1). Quando as reações foram realizadas apenas em THF, conforme procedimento descrito na literatura ${ }^{20}$, o composto (6) foi obtido em aproximadamente $70 \%$, enquanto o composto (5) não foi formado. Nesse último caso, a análise da mistura reacional por cromatografia em camada delgada revelou apenas a formação de uma mistura complexa. Os dados espectroscópicos do composto (6) foram idênticos àqueles relatados anteriormente ${ }^{20}$. Entretanto, no presente trabalho esse composto apresentou-se como um sólido branco cristalino com temperatura de fusão igual a $42-44{ }^{\circ} \mathrm{C}$, enquanto no trabalho mencionado ele apresentou-se como um óleo amarelo. Visando otimizar o rendimento dessas reações de alquilação, elas foram então realizadas em THF, na presença de N,N'-dimetil-N,N'-propileno uréia (DMPU) como co-solvente. Com esse procedimento os compostos (5) e (6) foram obtidos em rendimentos de $72 \%$ e $96 \%$, respectivamente. Em ambos os casos, as reações de alquilação foram estereosseletivas, sendo obtidos apenas os produtos exo-alquilados. Outro aspecto que deve ser notado é que, apesar de ter sido utilizada uma mistura racêmica do 2-bromopropionato de metila, foi obtido 


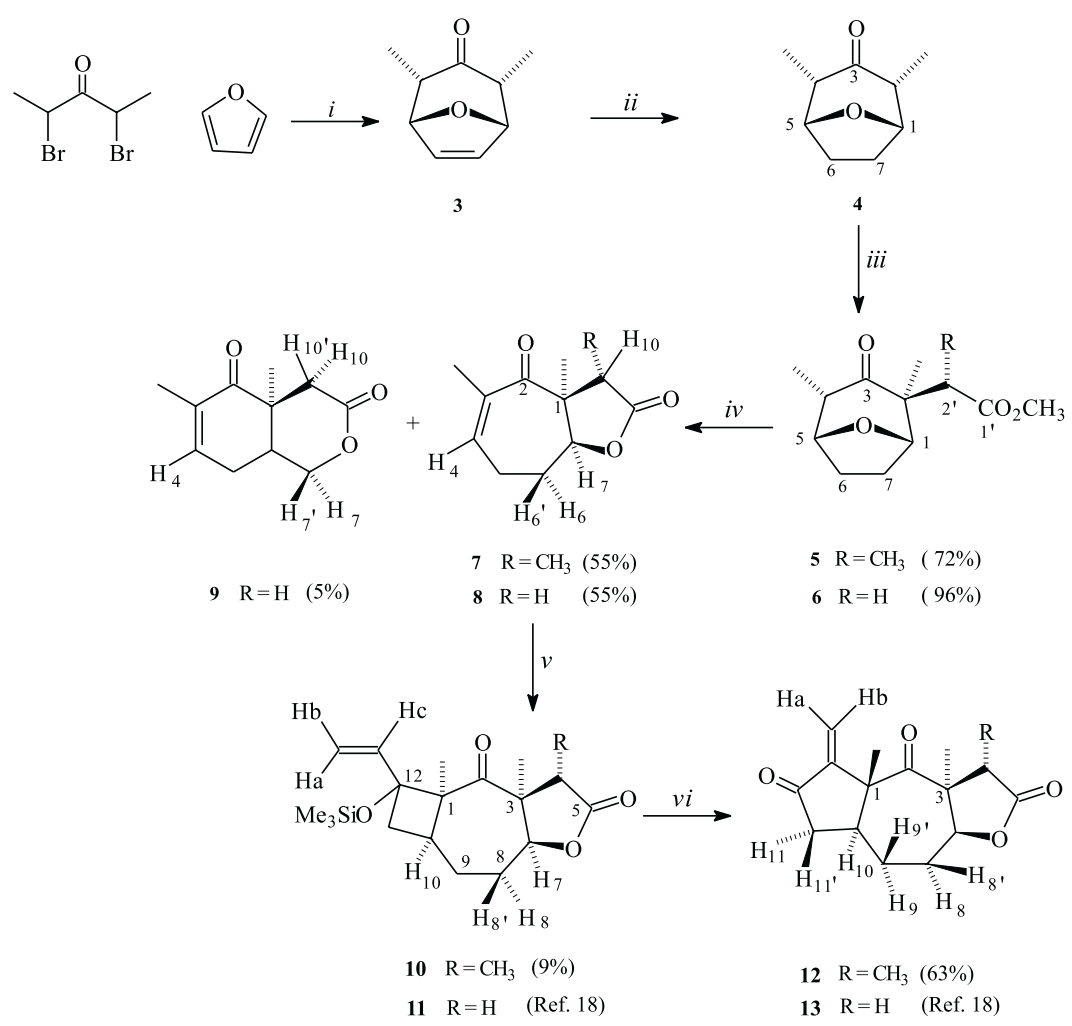

Esquema 1. i) $\mathrm{NaI}, \mathrm{Cu}, 60 \%$, ii) $\mathrm{AcOEt}, \mathrm{Pd} / \mathrm{C}$ (3 atm), 97\%; iii) $\mathrm{LDA}$, $\mathrm{THF}: \mathrm{DMPU}(2: 1),-78^{\circ} \mathrm{C}, 1,5 \mathrm{~h}$; $\mathrm{BrCHRCO}_{2} \mathrm{CH}_{3} ; 25^{\circ} \mathrm{C}, 16-18 \mathrm{~h}$; iv) $\mathrm{BF}_{3}, \mathrm{KI} \mathrm{CHCl}_{3}$, refluxo, 7-10 dias; v) $\mathrm{CH}_{2}=\mathrm{CH}\left(\mathrm{OSiMe}_{3}\right)=\mathrm{CH}_{2}$, dietoxietano, $20^{\circ} \mathrm{C}, \mathrm{hv}$; vi) $\mathrm{Pd}(\mathrm{PhCN})_{2} \mathrm{Cl}_{2}$, p-benzoquinona, $\mathrm{THF}$, refluxo, $4 \mathrm{~h}$

apenas o isômero com o grupo metila da cadeia lateral (carbono $\mathrm{C}^{\prime}$ ') na face $\alpha$. A estereoquímica do carbono C2' de (5) (Esquema 1), não foi determinada diretamente a partir de sua estrutura, mas pelos dados de nOe obtidos para a lactona correspondente (7), onde não se observou intensificação do sinal de H7 mediante irradiação de H10.

A estereosseletividade, nesse caso, pode ser compreendida através da análise dos diferentes possíveis estados de transição originados a partir dos enolatos (A) e (B), conforme apresentados no Esquema 2 . Os estados de transição $5 \mathrm{a}^{\ddagger}$ e $5 \mathrm{~d}^{\ddagger}$ que resultariam na formação dos isômeros 5 a e 5 d, respectivamente, com o grupo metila na face $\beta$, devem ser instáveis devido à interação repulsiva entre o grupo metila do agente alquilante (isômero $R$ e $S$ ) e o par de elétrons não-ligantes do oxigênio $\mathrm{sp}^{3}$. Por outro lado, os estados de transição $5 b^{\ddagger}$ e $5^{\ddagger}$, que resultam nos enantiômeros 5 b e (5), respectivamente, devem ser estáveis pois, nesse caso, o par de elétrons não-ligantes do oxigênio $\mathrm{sp}^{3}$ estaria direcionado para o átomo de hidrogênio do agente alquilante (isômero $R$ ou $S$ ). Embora o Esquema 1 ilustre a rota sintética com apenas um dos enantiômeros para os compostos (5) e (6), deve ficar claro nesse ponto que os compostos obtidos a partir de (5) e (6) também foram obtidos como mistura racêmica.

A clivagem da ligação C-O-C do grupo éter de (6), seguida de lactonização para a formação de (8), foi realizada anteriormente em presença de iodeto de trimetilsilano e também por meio do tratamento com $\mathrm{BF}_{3} / \mathrm{KI}^{20}$. Apesar da literatura relatar que o material de partida (6) foi consumido totalmente após três dias de reação $\operatorname{com} \mathrm{BF}_{3} / \mathrm{KI}$, no presente caso, necessitou-se de sete dias para que a reação se completasse. Outro aspecto importante é que, juntamente com a lactona (8), foi isolado também o composto (9) em 5\% de rendimento.

A fórmula molecular $\mathrm{C}_{11} \mathrm{H}_{14} \mathrm{O}_{3}$ para o composto (9) foi deduzida pela análise elementar. $\mathrm{O}$ espectro na região do infravermelho de (9) apresentou uma banda de absorção em $1737 \mathrm{~cm}^{-1}$ consistente com a presença da lactona de seis membros. A banda $1670 \mathrm{~cm}^{-1}$ indicou a presença do grupo carbonila $\alpha, \beta$-insaturado. O espectro de $\mathrm{RMN}$ de ${ }^{13} \mathrm{C}$ para esse composto apresentou 11 sinais, compatíveis com a estrutura proposta. Os sinais em $\delta 199,5$ e $\delta 168,5$ foram atribuídos às carbonilas da lactona e da cetona, respectivamente. O espectro de RMN de ${ }^{1} \mathrm{H}$ apresentou um multipleto em $\delta 6,60$, atribuído ao hidrogênio vinílico $\mathrm{H} 4$, e dois dupletos duplos em $\delta 4,18$ e $\delta 4,58$, que foram atribuídos aos hidrogênios metilênicos $\mathrm{H} 7$ e H7', respectivamente.

$\mathrm{O}$ tratamento do composto $(\mathbf{5})$ com $\mathrm{BF}_{3} / \mathrm{KI}$ resultou na obtenção da lactona (7) com rendimento de 55\%. A análise elementar do composto (7) foi consistente com a fórmula molecular $\mathrm{C}_{12} \mathrm{H}_{16} \mathrm{O}_{3}$. A unidade enona e o anel $\gamma$-lactona de (7) foram confirmados no espectro na região do infravermelho pela presença de absorções em $1677 \mathrm{~cm}^{-1}$ e $1767 \mathrm{~cm}^{-1}$, respectivamente. O espectro de RMN de ${ }^{1} \mathrm{H}$ mostrou sinais em conformidade com a estrutura proposta para (7), sendo o multipleto em $\delta 6,15$ atribuído ao hidrogênio vinílico H4. A estereoquímica desse composto foi confirmada a partir dos dados de nOe obtidos. A irradiação da metila em C-1 resultou em $2 \%$ de intensificação no sinal de $\mathrm{H} 7$, confirmando que a lactona apresenta estereoquímica cis. A irradiação de H10 não resultou em qualquer efeito sobre o sinal de $\mathrm{H} 7$, o que confirma que a metila ligada ao carbono C10 encontra-se na face $\alpha$.

A conversão da lactona (8) em (13), tendo como intermediário o produto de fotocicloadição (11), foi anteriormente descrita ${ }^{18}$. Neste caso, o intermediário (11) foi obtido com rendimento de 54\%, sendo então convertido na lactona correspondente (13) em $92 \%$ de rendimento.

Analogamente, a lactona tricíclica (12) também foi obtida pela metodologia de fotocicloadição descrita para o preparo de $(\mathbf{1 3})^{18,21}$, tendo como intermediário o composto (10). Entretanto, ao se comparar os rendimentos dos derivados sintetizados a partir de (7) e (8), nota-se que a natureza do substituinte $\mathrm{R}$ exerce uma significativa influência no rendimento das etapas seguintes, que levaram à obtenção das lactonas (12) e (13). Para $\mathrm{R}=\mathrm{H}$, a etapa de fotocicloadição 

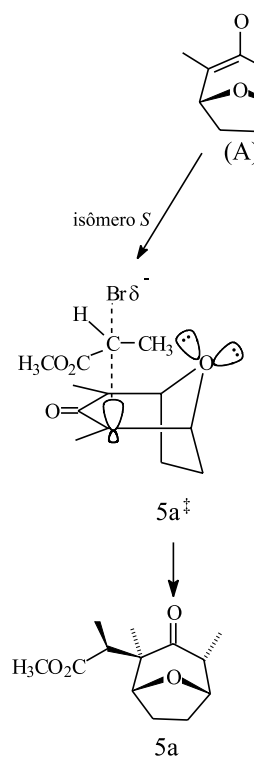
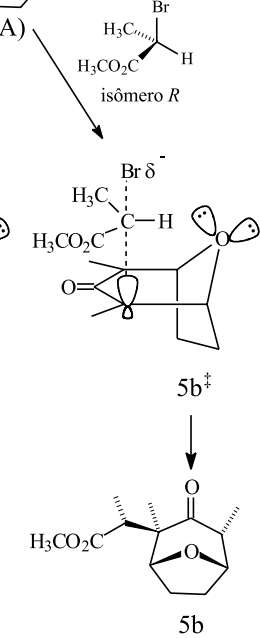

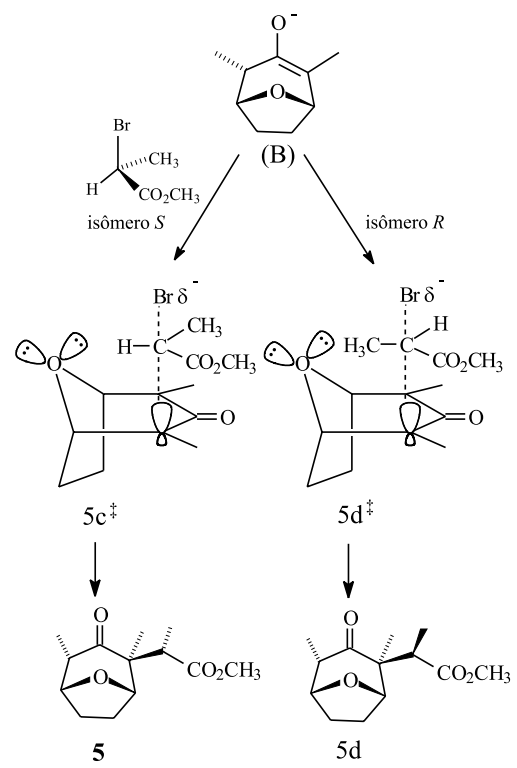

Esquema 2 forneceu o composto (11) com rendimento de $54 \%$, rendimento superior ao observado para $(\mathbf{1 0})\left(\mathrm{R}=\mathrm{CH}_{3}, 9 \%\right)$. Do mesmo modo, o rendimento da reação de expansão do anel ciclobutano de (11) para fornecer a lactona $(\mathbf{1 3})(\mathrm{R}=\mathrm{H}, 92 \%)$, foi superior ao rendimento para o caso de $(\mathbf{1 0})\left(\mathrm{R}=\mathrm{CH}_{3}, 63 \%\right)$, que levou à lactona $(\mathbf{1 2})^{18}$.

A análise elementar de (10) mostrou-se de acordo com a fórmula molecular $\mathrm{C}_{19} \mathrm{H}_{30} \mathrm{O}_{4} \mathrm{Si}$. O espectro de massas mostrou sinal do íon molecular em m/z 350,1913, também em concordância com o valor requerido. $\mathrm{O}$ espectro de $\mathrm{RMN}$ de ${ }^{1} \mathrm{H}$ de $(\mathbf{1 0})$ mostrou um sinal simples em $\delta 0,10$, que foi atribuído aos nove hidrogênios metílicos do grupo $\mathrm{O}-\mathrm{Si}(\mathrm{Me})_{3}$. Para os hidrogênios $\mathrm{Ha}, \mathrm{Hb}$ e $\mathrm{Hc}$, foram observados três dupletos duplos em $\delta 5,98, \delta 5,18$ e $\delta 5,28$, respectivamente.

A fórmula molecular $\mathrm{C}_{16} \mathrm{H}_{20} \mathrm{O}_{4}$ para a lactona (12) foi confirmada no espectro de massas pela presença do sinal em m/z 276,1335, conforme requerido. Os três grupos carbonila de (12) foram identificados no espectro na região do infravermelho pela presença das absorções em $1768 \mathrm{~cm}^{-1}, 1729 \mathrm{~cm}^{-1}$ e $1692 \mathrm{~cm}^{-1}$. Os espectros de RMN de ${ }^{1} \mathrm{H}$ e RMN de ${ }^{13} \mathrm{C}$ mostraram sinais de acordo com a estrutura de (12). Os dois hidrogênios olefínicos $(\mathrm{Ha}$ e $\mathrm{Hb}$ ) apresentaram um sinal simples em $\delta 5,94$ e $\delta 5,34$, respectivamente. Os sinais simples em $\delta 1,21, \delta 1,24$ e $\delta 1,33$ caracterizaram os hidrogênios metílicos. $\mathrm{O}$ espectro de RMN de ${ }^{13} \mathrm{C}$ mostrou um total de dezesseis sinais, sendo os sinais em $\delta$ 212,3, $\delta$ 201,6 e $\delta$ 176,2 atribuídos aos três grupos carbonila presentes na estrutura de (12).

Todos os deslocamentos químicos em RMN de ${ }^{1} \mathrm{H}$ foram confirmados através de experimentos COSY. Informações adicionais obtidas através de espectros nOe diferencial permitiram também a confirmação da correta estereoquímica proposta para (12). A Figura 1 apresenta os dados de nOe obtidos para a lactona (12).

A estereoquímica cis do anel $\gamma$-lactona com o anel de sete membros foi demonstrada pela observação de nOe $(1,5 \%)$ em H7, quando a irradiação foi feita sobre os hidrogênios metílicos em C3. Também foi observado nOe com o grupo metila $\alpha$ à carbonila lactônica $(2,5 \%)$ e com H10 (2,5\%), mostrando também a relação cis entre eles. Intensificação do sinal de H10 (nOe de 13\%) foi observada quando se fez a irradiação sobre $\mathrm{H} 7$, confirmando-se novamente a estereoquímica cis do anel $\gamma$-lactona. Por outro lado, a irradiação sobre H10 não provocou aumento do sinal dos hidrogênios metílicos em C1, mostrando assim a estereoquímica trans entre ambos. A maior blindagem do hidrogênio $\mathrm{Hb}(\delta 5,34)$, em relação ao hidrogênio Ha

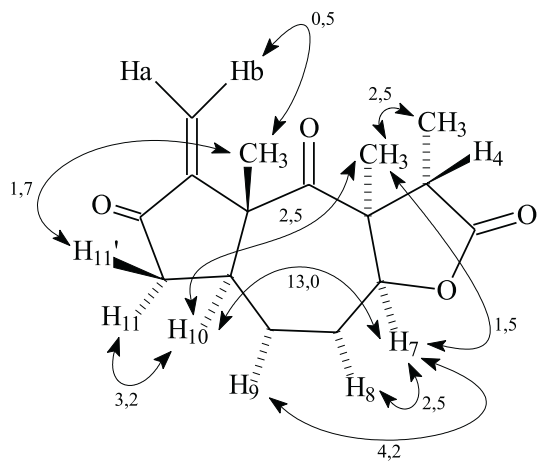

Figura 1. Principais dados de nOe (\%) para a lactona (12)

$(\delta 5,94)$, levou à observação de nOe $(0,5 \%)$ em $\mathrm{Hb}$, mediante irradiação sobre os hidrogênios metílicos em $\mathrm{C} 1$.

\section{Ensaios Biológicos}

Para a avaliação da atividade fitotóxica dos compostos sintetizados foram realizados ensaios utilizando-se Sorghum bicolor L. (sorgo, monocotiledônea) e Cucumis sativus L. (pepino, dicotiledônea). São plantas de crescimento rápido e que também permitem respostas visíveis nos ensaios ${ }^{3}$. Os resultados obtidos nos ensaios estão apresentados nas Figuras 2 e 3.

$\mathrm{O}$ "screening" aleatório é sabidamente uma prática de excelência que pode orientar para a busca de novos compostos com atividade herbicida ${ }^{22}$. Nesse sentido, os oxabiciclos (5) e (6), intermediários de reação, também foram submetidos aos testes para avaliação da atividade herbicida. Os resultados obtidos com esses compostos (5 e 6) mostraram baixas taxas de inibição sobre o crescimento radicular de sorgo e pepino. No ensaio com pepino observou-se indução do crescimento radicular, sendo o efeito mais pronunciado (67\%) quando se utilizou uma solução de (6) a $100 \mu \mathrm{g} \mathrm{mL}^{-1}$ (Figura 3).

A presença de grupos funcionais isoladamente em uma molécula não necessariamente lhe confere uma ação biológica expressiva. Há de se considerar a estrutura total da molécula para que se possa alcançar maior potenciação da atividade biológica. 


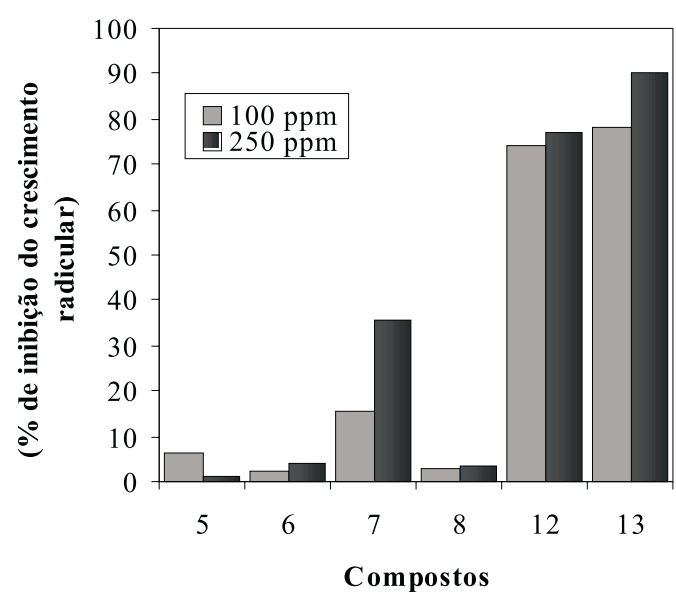

Figura 2. Efeito dos compostos (5), (6), (7), (8), (12) e (13) sobre o crescimento radicular de plantas de Sorghum bicolor $L$. (sorgo), nas concentrações de

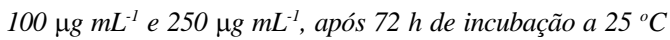

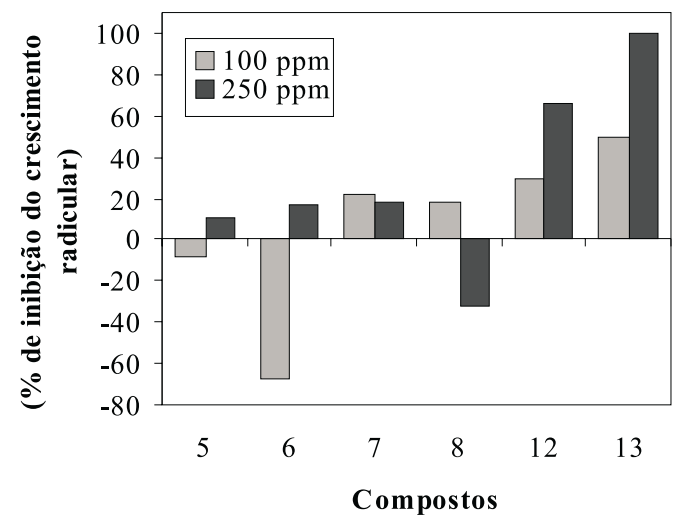

Figura 3. Efeito dos compostos (5), (6), (7), (8), (12) e (13) sobre o crescimento radicular de plantas de Cucumins sativus $L$. (pepino), nas concentrações de

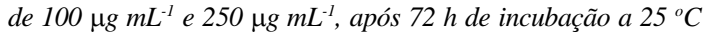

A presença do grupo carbonila $\alpha, \beta$-insaturado na estrutura das lactonas (7) e (8) refletiu em melhores resultados em relação aos compostos (5) e (6). Entretanto, resultados ainda pouco expressivos foram obtidos nos ensaios com essas lactonas, sendo a maior taxa de inibição $(35,8 \%)$ encontrada no ensaio com sorgo, quando se utilizou a lactona (7) a $250 \mu \mathrm{g} \mathrm{mL} \mathrm{m}^{-1}$. Também foi observada indução sobre o crescimento radicular de pepino para a lactona (8) a uma concentração de $250 \mu \mathrm{g} \mathrm{mL} \mathrm{m}^{-1}$.

Os resultados encontrados para as lactonas $(\mathbf{1 2})$ e (13), derivadas do 2,4-dimetil-8-oxabiciclo[3.2.1]-oct-6-en-3-ona (3), não diferiram significativamente entre si (Figuras 2 e 3). Entretanto, a posição ocupada pelo grupo metila no carbono C4 em (12) é estrategicamente importante, uma vez que sua conversão no grupo metileno pode resultar na potencialização da atividade biológica ${ }^{23}$.

A menor porcentagem de inibição sobre o crescimento radicular em sorgo foi de $74,1 \%$, quando se utilizou a solução $100 \mu \mathrm{g} \mathrm{mL}^{-1} \mathrm{de}$ (12), e a maior porcentagem (90\%) foi encontrada para a lactona (13), quando se utilizou a solução $250 \mu \mathrm{g} \mathrm{mL}^{-1}$. No ensaio com pepino foi encontrada porcentagem de inibição máxima (100\%) ao se utilizar a solução $250 \mu \mathrm{g} \mathrm{mL}^{-1} \mathrm{de}(\mathbf{1 3})$, e a menor porcentagem $(29,3 \%)$ foi observada para a solução $100 \mu \mathrm{g} \mathrm{mL}^{-1}$ de (12).

\section{CONCLUSÃO}

As lactonas (12) e (13), derivadas do 2,4-dimetil-8-oxabiciclo[3.2.1]oct-6-en-3-ona (3), foram obtidas em um total de seis etapas, partindose inicialmente de uma reação de cicloadição [3+4] entre 2,4dibromopentan-3-ona e furano. Considerando que a investigação do potencial fitotóxico de lactonas derivadas de oxabiciclos há muito aponta para a síntese de novas lactonas, este trabalho vem contribuir para a reunião de dados que possam conduzir à síntese de compostos com maior potencial de ação biológica. A lactona (13) mostrou $100 \%$ de inibição sobre pepino, na concentração de $250 \mu \mathrm{g} \mathrm{mL}^{-1}$, e a lactona (12) mostrou $90 \%$ de inibição sobre sorgo, na mesma concentração. Os resultados alcançados nesse trabalho e o sucesso da metodologia empregada estimulam o prosseguimento nas pesquisas para a busca de novos compostos com atividade herbicida. Objetivamos em nossos trabalhos realizar a conversão do grupo metila em C4 da lactona (12) em sua correspondente unidade $\alpha$-metileno, visando uma possível potencialização da atividade herbicida de (12). Portanto, a etapa da reação de cicloadição para o preparo de (10) deverá ser otimizada, uma vez que ela representa, no momento, um fator limitante para o preparo de (12).

\section{AGRADECIMENTOS}

Os autores agradecem ao Conselho Nacional de Desenvolvimento Científico e Tecnológico (CNPq) e à Fundação de Amparo à Pesquisa do Estado de Minas Gerais (FAPEMIG) pelo apoio financeiro.

\section{REFERÊNCIAS}

1. Copping, L. G.; Crop Protection Agents From Nature, Natural Products and Analogues, SCI: London, 1996, p. 501.

2. Godfrey, C. R. A.; Agrochemicals From Natural Products, $2^{\text {nd }}$ ed., Marcel Dekker: New York, 1994, p. 418.

3. Baruah, N. C.; Sarma, J. C.; Barua, N. C.; Sarma, S.; Sharma, R. P.; Phytochemistry 1994, 36, 29.

4. Macias, F. A.; Galindo, J. C. G.; Massanet, G. M.; Phytochemistry 1992, $31,1969$.

5. Arnason, J. T.; Isman, M. B.; Philogene, J. R.; Waddel, T. G.; J. Nat. Prod. 1987, 50, 690 .

6. Picman, A. K.; Biochem. Syst. Ecol. 1986, 14, 255.

7. Abad, M. J.; Bermejo, P.; Valverde, S.; Villar, A.; Planta Med. 1994, 60, 228.

8. Vichnewisk, W.; Gilbert, B.; Herz, W.; Phytochemistry 1976, 15, 191.

9. François, G.; Passreiter, C. M.; Wierdenbag, H; J.; Looveren, M. V.; Planta Med. 1996, 62, 126.

10. Macias, A. F.; Torres, A.; Molinillo, J. M. G.; Phytochemistry 1996, 43, 1205.

11. Demuner, A. J.; Barbosa, L C. A.; Piló-Veloso, D.; Quim. Nova 1997, 20, 18.

12. Costa, A. V.; Barbosa, L. C. A.; Demuner, A. J.; Silva, A. A.; J. Agric. Food Chem. 1999 47, 4807.

13. Barbosa, L. C. A.; Demuner, A. J.; Borges, E. E. L.; Mann, J; J. Braz. Chem. Soc. 1997, 8, 19.

14. Barbosa, L. C. A.; Conceição, G. A.; Demuner, A. J.; Silva, A. A.; Veloso, D. P.; Mann, J.; Aust. J. Chem. 1999, 52, 929.

15. Perrin, D. D.; Amarego, W. L.; Purification of Laboratory Chemicals, $3^{\text {rd }}$ ed., Butterworth-Heinemann Ltd.: Londres, 1988, p. 340.

16. Einhellig, F. A.; Schan, M. K.; Rasmunsem, J. A.; Plant Growth Regulators, 1983, p. 251.

17. Gomes, F. P.; Curso de Estatística Experimental, $3^{\text {rd }}$ ed., Nobel: Piracicaba, 1990, p. 404.

18. Barbosa, L. C. A.; Mann, J.; J. Chem. Soc. Perkin Trans 1 1992, 337.

19. Brown, C. A.; J. Am. Chem. Soc. 1969, 91, 5901.

20. Cummins, W. J.; Drew, M. G. B.; Mann, J.; Markson, A. J.; Tetrahedron 1988, 44, 5151 .

21. Demuth, M.; Pandley, B.; Wietfield, B.; Said, H.; Viader, J.; Helv. Chim. Acta 1988, 71, 1392.

22. Cobb, A.; Herbicides and Plant Physiology, Chapman \& Hall Limited: London 1992, p. 176.

23. Kupchan. S. M.; Fessler, D. C.; Eakin, M. A.; Giacobbe,T. J.; Science 1970, 168, 376. 